\title{
GEOMETRIC CIRCULAR GRAPHS
}

\author{
Medha Huilgol*
}

\section{ABSTRACT}

In this paper we study a class of graphs, which resemble a Circle in a plane in terms of diameter and radius. We introduce the term "Geometric circular graphs" for those graphs whose diameter is equal to twice the radius of the graph. Here we have studied some properties of geometric circular graphs. Also we have found some bounds in ferms of the number of edges.

\section{AMS Classification: 05C}

Key words and Phrases: Diameter and Radius of a graph.

\section{Introduction}

In this paper we introduce a class of graphs in which we bring the notion of a circle, along with radius and diameter, from Euclidian geometry. In literature, the concept of radius (and diameter) has been introduced on the basis of distance in graphs. It is well known the diameter of a circle is twice its radius in Euclidian geometry. But in case of graphs the diameter is bounded by radius at the lower end and twice radius of the graph on the upper end. Hence diameter assumes any value between these two extremes. The class of graphs for which radius is equal to

- Department of PG Mathematics, Christ College, Hosur Road, Bangalore, Medha_huilgol@yahoo.com. 
diameter has been studied by [1], [3], under the title "self-centered graphs" or "equi-eccentric graphs". The class of graphs for which diameter is twice the radius of a graph, exactly resembles circle in a plane. Hence we term these graphs as "Geometrical Circular Graphs", in short GCG.

Definition : A connected graph is said to be a geometric circular graph, GCG, if its diameter is twice radius, that is $\operatorname{diam}(G)=2 \operatorname{rad}(G)$.

For all definitions and terminology the reader may refer [4]. In particular, in this paper the diameter of a connected graph $G$ is denoted by $\operatorname{diam}(G)=d(G)$ and the radius of the connected $\operatorname{graph} G$ is denoted by $\operatorname{rad}(G)=r(G)$.

\section{Existing Results and Definitions}

In this section we list some existing results, without proof, and definitions, which are helpful in proving the results of this paper.

Proposition 1.1[5]: Suppose that all diametral paths of a graph $G$ avoid the center, then $r(G)+2 \leq d(G) \leq 2 r(G)-1$ and all pairs of values in this given range are attainable.

Proposition 1.2[6]: If $d(G) \geq 3$, then $d(\bar{G}) \leq 3$.

Proposition 1.3[4]: Every non-trivial self-complementary graph has diameter 2 or 3 .

Proposition 1.4[4]: Any graph $\mathrm{G}$ can be embedded in a supergraph $\mathrm{H}$ such that $<\mathrm{C}(H)\rangle$ is isomorphic to $\mathrm{G}$.

Proposition 1.5 [8]: Let $\mathrm{G}$ and $\mathrm{H}$ be simple connected graphs. Then

$r(G \times H)=r(G)+r(H)$

$d(G \times H)=d(G)+d(H)$

Proposition 1.6[9]: For a graph $G$ if $d(G)=2 r(G)$, then $P(G) \subseteq E C(G)$.

\section{Results}

In this section we prove some results on geometric circular graphs. 
Lemma 2.1: A connected graph is geometric circular graph, GCG, if at least one diametral path contains a central vertex.

Proof: Let $G$ be a connected graph. By Proposition 1.1 [5], if all diametral paths avoid the center, then $d(G) \leq 2 r(G)-1$, hoids. If at least one diametral path contains a central vertex, then $d(G) \geq 2 r(G)$. Since $G$ is connected $d(G) \leq 2 r(G)$. Hence equality holds, making the graph $G$ to be a GCG.

Proposition 2.2: If $G$ is a $G C G$, then any diametral path cannot contain more than one central vertex.

Proof: For a GCG, G, let $x-y$ be a diametral path. Suppose the $x-y$ diametral path contains two central vertices, viz. $u$ and $v$. Then,

$d(x, y) \leq d(x, u)+d(u, y)+d(u, v)$

$\Rightarrow d(x, y)=d(G) \leq 2 r(G)+d(u, v) \Rightarrow 2 r(G) \leq 2 r(G)+d(u, v) \Rightarrow d(u, v)=0 \Rightarrow u=v$.

Hence there lies exactly one central vertex on any diametral path.

Note: Clearly, if $G$ is a GCG, then there does not exist a diametral path containing an edge of $\langle C(G)\rangle$.

Proposition 2.3: In a GCG, every central vertex has at least two eccentric vertices.

Proof: Let $G$ be a GCG. Let $x$ be a central vertex of $G$. By Proposition 2.2 above, $x$ lies on a diametral path, say, $u$-v path, i.e. $d(u, v)=d(G)=2 r(G)$. If $x$ is a midpoint of $u-v$ path, then $u, v$ will be the eccentric vertices of $x$.

If $x$ is not a midpoint of $u-v$ path, then, for the midpoint of $u-v$ path, say, $x^{\prime}, e\left(x^{\prime}\right) \geq \frac{1}{2} d(G)=r(G)$

If $e\left(x^{\prime}\right)>\frac{1}{2} d(G)$, then $x^{\prime} \notin C(G)$, where $C(G)$ denotes the center of the graph $G$.

If $e\left(x^{\prime}\right)=\frac{1}{2} d(G)$, then $x^{\prime}(=x) \in C(G)$. Hence, $x^{\prime}$ will have $u$ and $v$ as its eccentric vertices.

Note: From the above result it is clear that every central vertex is a midpoint of some diametral path in a GCG. But the converse need not always be true. 
Theorem 2.4: A connected graph $G$ is $G C G$ if and only if $P(G)=E C(G)$, where $P(G)$ denotes the periphery of $G$ and $E C(G)$ denotes the set of all eccentric vertices of central vertices of $G$.

Proof: Let $G$ be GCG. By Proposition 1.6 [9] , $P(G) \subseteq E C(G)$.

Conversely, if $x \in E C(G) \Rightarrow$ there exists a vertex $u \in C(G)$ such that $d(x, u)=r(G)$ and $x$ is an eccentric vertex of $u$ i.e. $d(x, u)=1 / 2 d(G)$. By the above proposition there exists at least one more eccentric vertex for $u$, say, $y$. Hence, $d(x, y)=d(x, u)$ $+d(u, y)=2 r(G)=d(G)$. Hence, $x, y \in P(G)$.

So $E C(G) \subseteq P(G)$, making the two sets equal in case of geometric circular graphs.

Proposition 2.5: If a graph $G$ is $G C G$, then its complement $\bar{G}$ is not.

Proof: Let $G$ be a GCG. Hence, $G$ is connected and has finite radius and diameter. If $d(G) \geq 3$, then by Proporosition 1.2[6], $d(\bar{G}) \leq 3$ If possible assume that $\bar{G}$ is $G C G$, then $d(\bar{G})=2$ and $r(\bar{G})=1$. Hence, in $\bar{G}$ there exists a vertex, say, $u$, such that $\operatorname{deg}_{\bar{G}} v=p-1 \Rightarrow \operatorname{deg}_{G} v=0$, a contradiction to the connectedness of the graph $G$.

If $d(G)<3$ then $d(G) \leq 2$ and hence, $d(G)=2, r(G)=1$. Similar argument as above leads to the contradiction to the connectedness of $\bar{G}$, if it were GCG. Hence, if $G$ is $G C G$, then is not.

Note: If $\bar{G}$ is $G C G$, then $G$ is not a $G C G$.

Theorem 2.6: There does not exist a self-complementary GCG.

Proof: By above proposition the proof follows.

Proposition 2.7: Any connected graph $G$ can be embedded in some GCG.

Proof: Let $G$ be a connected graph. We know that it it is easy to embed $G$ as an induced subgraph in any supergraph $\mathrm{H}$ such that $\langle\mathrm{C}(\mathrm{H})\rangle$ is isomorphic to $\mathrm{G}$ by [4]. Indeed, it is the sequential join

$K_{1}+K_{1}+\ldots . .+K_{1}(r$-times $)+G+K_{1}+\ldots \ldots .+K_{1}(r$-times $)$. These graphs have $\operatorname{rad}(H)=r$ and $\operatorname{diam}(H)=2 r$, and hence these are geometric circular graphs.

Proposition 2.8: The Cartesian product of two graphs is GCG if, and only if both graphs are geometric circular. 
Proof: Let $G_{1}$ and $G_{2}$ be any two geometric circular graphs. Let $G=G_{1} \times G_{2}$ be the Cartesian product of $G_{1}$ and $G_{2}$. By Proposition 1.5[8], $r\left(G_{1} \times G_{2}\right)=r\left(G_{1}\right)+r\left(G_{1}\right)$ and $d\left(G_{1} \times G_{2}\right)=d\left(G_{1}\right)+d\left(G_{2}\right)$ Since both $G_{1}$ and $G_{2}$ are geometric circular, $d\left(G_{1}\right)=2 r\left(G_{1}\right)$ and $d\left(G_{2}\right)=2 r\left(G_{2}\right)$. Hence, $d\left(G_{1} \times G_{2}\right)=d\left(G_{1}\right)+d\left(G_{2}\right)=$ $2 r\left(G_{1}\right)+2 r\left(G_{2}\right)=2\left(\left[r\left(G_{1}\right)+r\left(G_{2}\right)\right]=2 r\left(G_{1} \times G_{2}\right)\right.$ making the Cartesian product to be a geometric circular graph.

For the converse, let the Cartesian product of two graphs be geometric circular, i.e. for any two graphs $G_{1}$ and $G_{2^{\prime}}$ let $G=G_{1} \times G_{2^{\prime}}$ be $G C G$. Hence, $d\left(G_{1} \times G_{2}\right)=$ $2 r\left(G_{1} \times G_{2}\right) \Rightarrow d\left(G_{1}\right)+d\left(G_{2}\right)=2 r\left(G_{1}\right)+2 r\left(G_{2}\right)$

$$
\Rightarrow r\left(G_{1}\right)=\frac{d\left(G_{1}\right)+d\left(G_{2}\right)-2 r\left(G_{2}\right)}{2}
$$

If $d\left(G_{2}\right)=2 r\left(G_{2}\right)$ that is if $G_{2}$ is $G C G$, then $G_{1}$ is also $G C G$.

If $d\left(G_{2}\right)<2 r\left(G_{2}\right)$, then $d\left(G_{1}\right)<2 r\left(G_{1}\right)$. Hence $d\left(G_{1} \times G_{2}\right)<2 r\left(G_{1} \times G_{2}\right)$, a contradiction to the assumption that $G=G_{1} \times G_{2}$ is a geometric circular graph.

So both $G_{1}$ and $G_{2}$ have to geometric circular if their Cartesian product is geometric circular.

Proposition 2.9: For a GCG, $G$, of order $p$, radius r, diameter $d$, size $q$, and the following holds true: $p-1 \leq q \leq \frac{(p-d)^{2}+3 p-d-4}{2}$, and both the bounds are attainable.

Proof: Given G is GCG. Hence d is finite, so we need at least p-1 edges to cover all vertices. Hence, the lower bound follows and a tree with one central vertex attains it.

For the upper bound, we have $d(G)=2 r(G)$. Hence there exist at least one diametral path of length $d$. Let $v_{1}, v_{2^{2}}, \ldots . ., v_{d}, v_{d+1}$, be one of the diametral paths. Let the vertex set $V(G)$ be partitioned into subsets $V_{1}, V_{2}, \ldots . ., V_{d}, V_{d+1}$ such that each $v_{i} \in V_{i}$ and all those vertices $x \in V_{i}$ satisfying the following two conditions: $d\left(x, v_{1}\right) \leq d$ and $d\left(x, v_{d+1}\right) \leq d$ otherwise the length of a path containing $x, v_{1}, v_{2^{\prime}}, \ldots ., v_{d^{\prime}}, v_{d+1}$ exceeds the diameter. This partition is possible because each $V_{i}$ contains at least one vertex viz., $v_{i}$ itself. We can add maximum possible edges to each $V_{i}$ among $v_{i}^{\prime}$ s and among vertices of $V_{i}$ and $V_{i+1}$, without disturbing $d(G)$. The resulting graph 
is $K_{n_{1}}+K_{n_{2}}+\ldots \ldots+K_{n_{d+1}}$, where $\sum_{i=1}^{d+1} n_{i}=p$ This graph is maximal with respect to the diameter in the sense that by adding an additional edge to the graph the diameter is decreased by one. Hence, $q=\sum_{i=1}^{d+1}\left(\begin{array}{l}n_{i} \\ 2\end{array}\right)+\sum_{i=1}^{d} n_{i} n_{i+1}$ So $q$ is maximum when both summands are maximum.

For $\sum_{i=1}^{d+1}\left(\begin{array}{l}n_{i} \\ 2\end{array}\right) \leq\left(\begin{array}{c}d+1 \\ \sum_{i=1} n_{i} \\ 2\end{array}\right)=\left(\begin{array}{c}p-d \\ 2\end{array}\right)$ as at least $d$-vertices are required to maintain the diameter.

But sum of the products $\sum_{i=1}^{d} n_{i} n_{i+1}$ is maximum whenever $n_{i}=n_{i+1}$.

As $p$-d vertices have already gone in first term there remain only $d$-vertices in second term. So, $G$ would be isomorphic to $K_{p-d}+K_{1}+K_{1}+\ldots . .+K_{1}(d-$-imes $)$.

The size of the graph is maximum if

$$
\begin{aligned}
& G \cong K_{1}+K_{1}+\ldots . .+K_{1}(r-\text { times })+K_{p-d}+K_{1}+K_{1}+\ldots . .+K_{1}(r-\text { times }) \text {. So, } \\
& q \leq\left(\begin{array}{c}
p-d \\
2
\end{array}\right)+2(p-d)+2(r-1)=\frac{(p-d)(p-d-1)+4(p-d)+4(r-1)}{2} \\
& q \leq \frac{(p-d)(p-d-1+4)+(2 d-4)}{2}=\frac{(p-d)^{2}+3 p-d-4}{2} .
\end{aligned}
$$

Hence, $G \cong K_{1}+K_{1}+\ldots . .+K_{1}(r$-times $)+K_{\rho . d}+K_{1}+K_{1}+\ldots . .+K_{1}(r$-times $)$ is the realizing graph for the upper bound of $q$.

In Theorem 1.6[9], it is proved that if a graph $G$ has $d(G)=2 r(G)$, i.e. if the graph $G$ is $G C G$, then $P(G) \subseteq E C(G)$.In view of this result one may consider the size of the sets $P(G)$ and $E C(G)$. The following proposition gives a graph with given size of $P(G)$ and $E C(G)$.

Proposition 2.10: For any positive integers $m$ and $n, 2 \leq m \leq n$, there exists a geometric circular graph $G$ such that $|P(G)|=m,|E C(G)|=n$. 
Proof: Consider a path $P_{2 r}: v_{1}, v_{2}, \ldots ., v_{r+1}, v_{r+2}, \ldots, v_{2 r+1}$. And consider another path of length $r$ whose end vertex is concatenated with $v_{r+1}$, and it is labeled as $v_{r+1}, u_{1}, u_{2}, \ldots \ldots, u_{r}$. Form a graph $G$ by joining $\mathrm{m}-2$ end vertices with $v_{2}$ and $n-m-1$ end vertices with $u_{r .1}$. Labeling these extra end vertices in forming $G$ as $w_{1}, w_{2}, \ldots, w_{m-2^{\prime}}$ and $w^{\prime}{ }_{1}, w_{2}^{\prime}, \ldots, w_{n \cdot m-l}^{\prime}$. Clearly, $d(G)=2 r(G)$. Hence $G$ is a GCG.

Also it is not difficult to observe that $P(G)=\left\{v_{1}, w_{1}, w_{2}, \ldots . ., w_{m \cdot 2}, v_{2 r+1}\right\}$ and $E C(G)=\left\{v_{r^{\prime}}, w^{\prime}{ }_{1}, w_{2^{\prime}}^{\prime} \ldots . ., w_{n \cdot m \cdot\}}^{\prime}\right\} \cup P(G)$.

Therefore $|P(G)|=m,|E C(G)|=m+n-m-1+1=n$. Hence the result.

\section{References}

1. J. Akiyama, K. Ando and D. Avis, "Miscellaneous properties of equi-eccentric graphs", Convexity and Graph Theory, Proc. Conf. in Haifa, Israel, 1981, North.Holland, Amsterdam (1984)13-23.

2. J.C. Bermond and B. Bollabas, "The diameter of graphs-a survey", Congressus Numer. 23(1981)3-27.

3. F. Buckley, "Self-centered graphs with a given radius" Congressus Numer., 23(1979) 211-215.

4. F. Buckley and F. Harary, "Distance in Graphs" Addison-Wesley Publishing Company, 1990.

5. F. Buckley and M. Lewinter, "Minimal graph embeddings, eccentric vertices and the peripherian", Proceedings of the Fifth Caribbean Conference on Combinatorics and Computing, University of the West Indies (1988) 72-84.

6. F. Harary and R.W. Robinson, "The diameter of a graph and its complement", Amer. Math. Monthly. 92 (1985) $211-212$.

7. R. Nadakumar and K.R. Parthasarathy, "Unique eccentric point graphs", Discrete Math.46 (1983) 69-74.

8. J. Niemien, "The Center Problem in the Product of graphs", Recent Studies in Graph Theory, ed. V.R. Kulli, 1989; Vishwa Intemational Publications, 201-205.

9. K.B. Reid and W. Gu,: "Peripheral and Eccentric vertices in Graphs", Graphs and Combinatorics(1982)8:361-375. 